Gesnerus 54 (1997) 330-335

\title{
Contents of Vol. 54 (1997)
}

\section{Original Articles}

Dasen, Véronique: Autour de l'estropié du Musée d'art et d'histoire de Genève: une représentation archaïque grecque d'hémimélie? [The Cripple of the Geneva Museum of Art and History: An Archaic Greek Representation of Hemimelia?] ..............

Diosi, Peter: Albert Jesionek auf der Spur der Zytomegalie-Krankheit [Albert Jesionek on the Track of Cytomegalia]

Gasser, Jacques; Heller, Geneviève: Étude de cas: les débuts de la stérilisation légale des malades mentaux dans le canton de Vaud [Case Study: The Beginning of Legal Sterilization of Mentally Ill Persons in the Swiss Canton Vaud] $\ldots \ldots \ldots \ldots \ldots \ldots \ldots \ldots \ldots$.

Keel, Othmar; Hudon, Philippe: L'essor de la pratique clinique dans les armées européennes (1750-1800) [The Progress of Clinical Practice in the European Armies $(1750-1800)]$

Kiehne, Tilman: Die Promotionsarbeit des Joseph von Quarin als Spiegel des Standes der medizinischen Entomologie in der Mitte des 18. Jahrhunderts [Joseph von Quarin's Doctoral Thesis as a Mirror of the State of Medical Entomology in mid-18 ${ }^{\text {th }}$ Century] ......

Koelbing, Huldrych M.: Englisch - die universelle Wissenschaftssprache des 20. Jahrhunderts. Alphonse de Candolle's Prognose von 1873 [English as Universal Language of Science in the $20^{\text {th }}$ Century. Alphonse de Candolle's Prognosis of 1873$] \ldots \ldots \ldots \ldots$.

Lohff, Brigitte: Die Rezeption der Werke Johann Georg Zimmermanns in Montpellier [The Reception of Johann Georg Zimmermann's Works in Montpellier] ............

Premuda, Loris: Padua und die Medizin [Medicine in Padua] ...................

Prüll, Cay-Rüdiger: Von «grossen Deutschen» und «stolzen Wipfeln»- Die Lehrbücher der deutschen Medizingeschichte und die Pathologie (1858 bis 1945) [Pathology in the

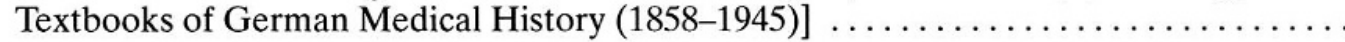

Rieder, Philip: Entre liberté et contrôle, les privat-docents et l'enseignement de la médecine à Genève entre 1876 et 1917 [Between Freedom and Supervision, the privat-docents and Medical Teaching in Geneva between 1876 and 1917] .....................

Schüppel, Reinhart: Die Bedeutung kognitiver Prozesse in der Arzneimittelforschung im 19. Jahrhundert - Das Beispiel Nitroglycerin [Cognitive Processes in the 19th Century Drug Research: The Example of Nitroglycerine] 
Wiesing, Urban: Die Einsamkeit des Arztes und der «lebendige Drang nach Geschichte». Zum historischen Selbstverständnis der Medizin bei Richard Koch [The Physicians's Loneliness and the «desire for history». Richard Koch and the Historical View of Medicine]

\section{Short Communication}

Bäschlin, Daniel L.: Zum rätselhaften Bild des Kosmos bei Camille Flammarion [Camille Flammarion's Enigmatic Image of the Universe $] \ldots \ldots \ldots \ldots \ldots \ldots \ldots \ldots \ldots$

News and Activities

Books Received

\section{Book Reviews}

Essay review: Geiser, Marc: Apparition, évolution et fin(?) du concept de schizophrénie

Antike Naturwissenschaft und ihre Rezeption (Schulthess)

Ärztelexikon (Ritzmann)

Avogadro, Amedeo: Saggi e memorie sulla teoria atomica (1811-1838) (Felder-Casa-

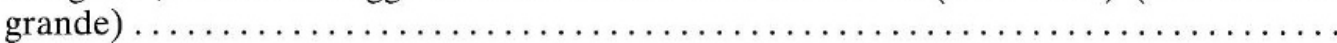

Beretta, Marco: Il tesoro della salute. Dall'onnipotenza dei semplici all'atomizzazione del farmaco (Ledermann)

Binneveld, Hans: Om de geest van Jan Soldaat (Blok)

Bonnet: Charles Bonnet, savant et philosophe (1720-1793) (Boschung)

Bono, James J.: The word of God and the languages of man (Maclean)

Brinkschulte, Eva (Hrsg.): Weibliche Ärzte (Müller-Landgraf)

Bröer, Ralf: Salomon Reisel (1625-1701). Barocke Naturforschung eines Leibarztes im

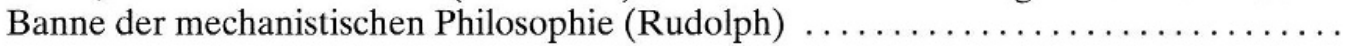

Capponi, Filippo: L'anatomia e la fisiologia di Plinio (Zimmermann)

Carozzi, Albert V.; Newman, John K.: Horace-Bénédict de Saussure: forerunner in gla-

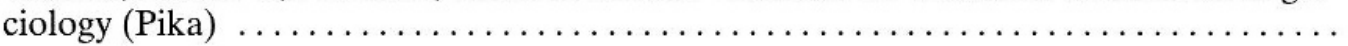

Chapman, Carleton B.: Order out of chaos. John Shaw Billings and America's coming of

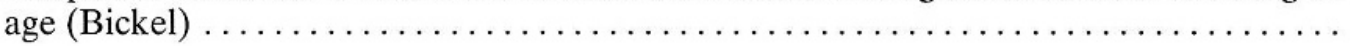

La correspondencia entre A. von Haller y Antonio Capdevila (Boschung)

Dinges, Martin (Hrsg.): Medizinkritische Bewegungen im Deutschen Reich (ca. 1870 -

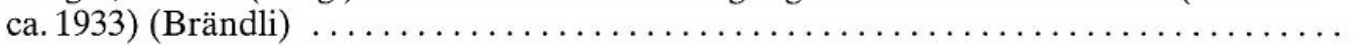

Eckart, Wolfgang U.; Gradmann, Christoph (Hrsg.): Die Medizin und der Erste Weltkrieg (Mörgeli) 
Eckart, Wolfgang U.; Volkert, Klaus (Hrsg.): Hermann von Helmholtz (Kauertz) ......

Einstein, Albert:The collected papers of Albert Einstein, vol.6: The Berlin years: writings, 1914-1917 (Straumann) . . . . . . . . . . . . . . . . . . . . . . . . . . .

Epple, Ruedi; Schnyder, Albert: Wandel und Anpassung. Die Landwirtschaft des

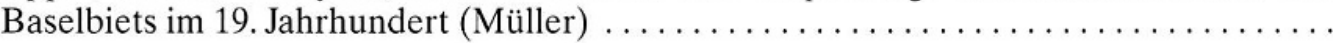

Euler, Leonhard: Commentationes mechanicae et astronomicae ad physicam cosmicam

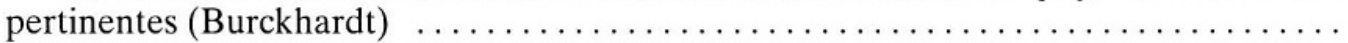

Fee, Elizabeth; Brown, Theodore M. (eds.): Making medical history. The life and times

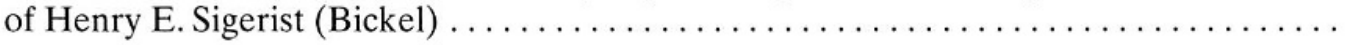

Fischer, Ernst Peter: Einstein: Ein Genie und sein überfordertes Publikum (Meyer) . . 288

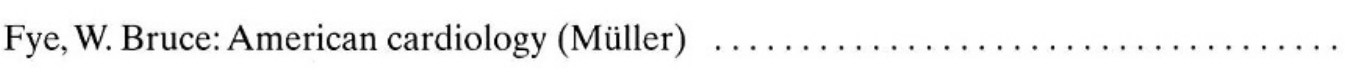

Gantenbein, Urs Leo: Schwitzkur und Angstschweiss (Suter)

Geschlechterverhältnisse in Medizin, Naturwissenschaft und Technik (Fischer-Hom-

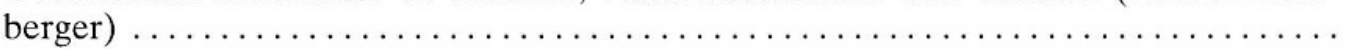

Gill, Glenys; Klenke, Dagmar: Institute im Bild, Teil 1: Bauten der Kaiser-WilhelmGesellschaft zur Förderung der Wissenschaften (Glaus) $\ldots \ldots \ldots \ldots \ldots \ldots \ldots$

Gloy, Karen: Das Verständnis der Natur. Zweiter Band: Die Geschichte des ganzheit-

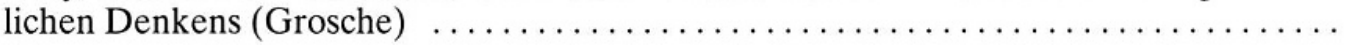

Goerke, Heinz: Am Puls der Medizin. Arzt im 20. Jahrhundert (Mörgeli) . ...........

Gossweiler, Margot: Regula Wolf-Gossweiler an Lisette Wolf. Briefe aus den Jahren

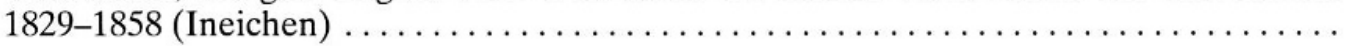

Gossweiler, Margot: Johannes Wild und Lisette Wolf. Briefwechsel aus den Jahren

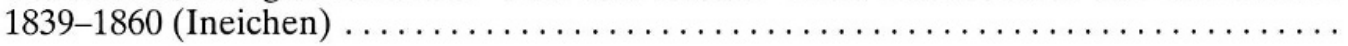

Grosche, Stefan: Lebenskunst und Heilkunde bei C.G. Carus (1789-1869). Anthropologische Medizin in Goethescher Weltanschaung (Scholz) .....................

Haskell, Francis: Die Geschichte und ihre Bilder (Neumann)

Hauke, Petra: Bibliographie zur Geschichte der Kaiser-Wilhelm-/Max-Planck-Gesellschaft zur Förderung der Wissenschaften (1911-1994) (Glaus) ...................

Heilemann, Hubert Gottfried: Goethe. Eine Krankengeschichte und kritische Darstel-

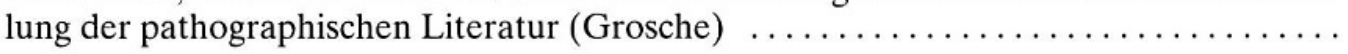

Herbst, Klaus-Dieter: Die Entwicklung des Meridiankreises 1700-1850 (Oechslin) . ...

Herzog, Eva: «Frisch, frank, fröhlich, frau» (Witzig) $\ldots \ldots \ldots \ldots \ldots \ldots \ldots \ldots \ldots$

Hoffmann-Axthelm, Walter: Geschichte der Mund-, Kiefer- und Gesichtschirurgie

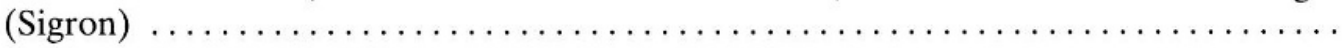

Holzhey, Helmut; Boschung, Urs (Hrsg.): Gesundheit und Krankheit im 18. Jahrhundert

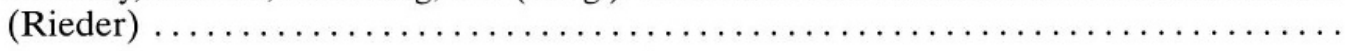




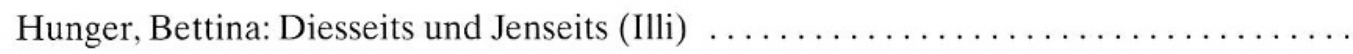

Hurt, Raymond: The history of cardiothoracic surgery from early times (Naef) ........

Immunology: the making of a modern science (Lindenmann) $\ldots \ldots \ldots \ldots \ldots \ldots \ldots$

Jayawardene, S. A.: The scientific revolution (Seger) $\ldots \ldots \ldots \ldots \ldots \ldots \ldots \ldots \ldots$

Jütte, Robert: Geschichte der Alternativen Medizin (Grosche)

Kaufmann, Doris: Aufklärung, bürgerliche Selbsterfahrung und die «Erfindung» der

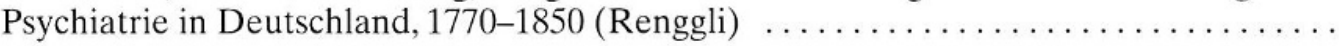

Kersting, Franz-Werner: Anstaltsärzte zwischen Kaiserreich und Bundesrepublik (Mül-

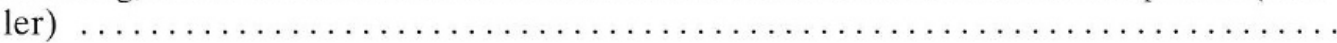

Kinzelbach, Annemarie: Gesundbleiben, Krankwerden, Armsein in der frühneuzeitlichen

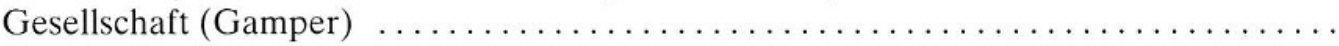

Krafft, Fritz (Hrsg.): «... der Himmel bewahre Sie vor einer socialistischen Herrschaft!»

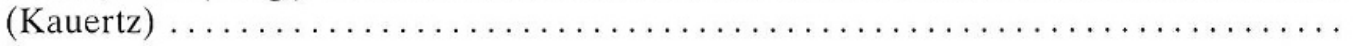

Kranke und Krankheiten im Juliusspital zu Würzburg 1819-1829 (Gerabek) . . . . . . . 136

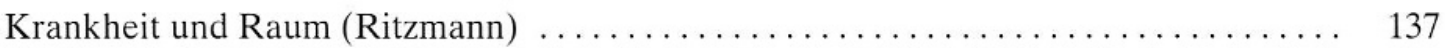

Krietsch, Peter; Dietel, Manfred: Pathologisch-Anatomisches Cabinet (Grosche) ..... 138

Kruse, Britta-Juliane: Verborgene Heilkünste. Geschichte der Frauenmedizin im Spät-

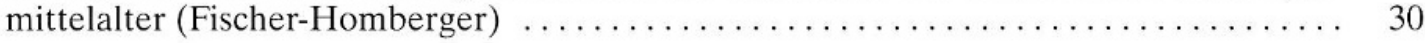

Kuhn, Karl: Medizinhistorische Institutionen und Publikationen (Seger) . . . . . . . . 139

Landriani, Marsilio: Ricerche fisiche intorno alla salubrità dell'aria (Felder-Casagrande) 302

Lea, Elisabeth; Wiemers, Gerald: Planung und Entstehung der Sächsischen Akademie der Wissenschaften zu Leipzig 1704-1846 (Glaus) .........................

Leibniz, Gottfried Wilhelm: Mathematischer, naturwissenschaftlicher und technischer Briefwechsel, 4. Band: Juli 1663-1690 (Fellmann) ..........................

Leonhardt, Martin: Hermann F. Hoffmann (1891-1944). Die Tübinger Psychiatrie auf dem Weg in den Nationalsozialismus (Renggli) ..........................

Löneke, Regina; Spieker, Ira (Hrsg.): Reinliche Leiber - Schmutzige Geschäfte (Müller-

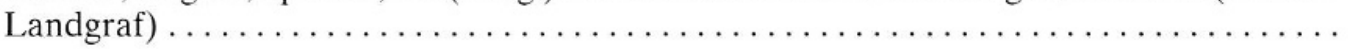

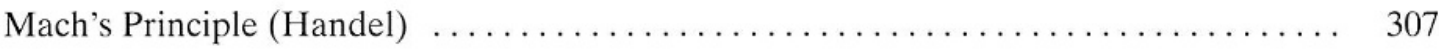

Marcovecchio, Enrico: Dizionario etimologico storico dei termini medici (Mudry) .....

Mazumdar, Pauline M. H.: Species and Specificity. An interpretation of the History of

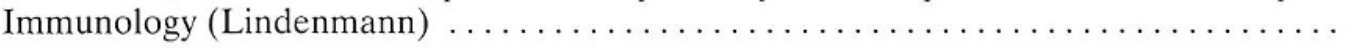

Meitzner, Bettina: Die Gerätschaft der chymischen Kunst (Gantenbein) … . . . . . 309

Mörgeli, Christoph: Europas Medizin im Biedermeier (Kanz) $\ldots \ldots \ldots \ldots \ldots \ldots \ldots$. 
Müller, Annette: Krankheitsbilder im Liber de Plantis der Hildegard von Bingen

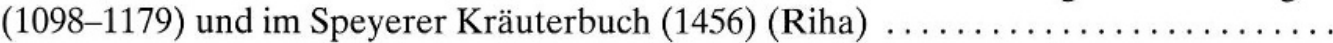

Müller-Jahncke, Wolf-Dieter; Friedrich, Christoph; Paulus, Julian (Mitarb.): Geschichte der Arzneimitteltherapie (Ledermann) . . . . . . . . . . . . . . . . . . . . . . .

Nutton, Vivian; Porter, Roy (eds.): The History of Medical Education in Britain (Rieder)

Othenin-Girard, Mireille: Ländliche Lebensweise und Lebensformen im Spätmittelalter (Ritzmann)

Paradigms and mathematics (Ineichen)

Parthey, Heinrich: Bibliometrische Profile von Instituten der Kaiser-Wilhelm-Gesellschaft zur Förderung der Wissenschaften (1923-1943) (Glaus) . . . . . . . . . . . . . . .

Paul, Diane B.: Controlling human heredity, 1865 to the present (Lindenmann) .......

Pichot, André: Die Geburt der Wissenschaft. Von den Babyloniern zu den frühen Grie-

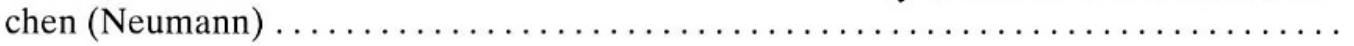

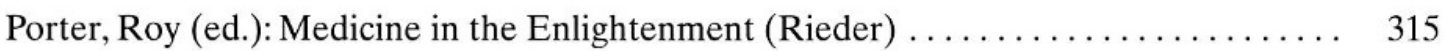

Psychoanalyse in Frankfurt am Main (Müller) ........................ 142

Regin, Cornelia: Selbsthilfe und Gesundheitspolitik (Blum) ............... 316

Roer, Dorothee; Henkel, Dieter (Hrsg.): Psychiatrie im Faschismus. Die Anstalt Hada-

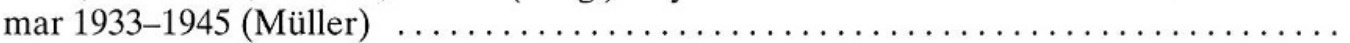

Scheifele, Irene: Festschriften der Versammlungen Deutscher Naturforscher und Ärzte 1822 bis 1920 (Glaus) . . . . . . . . . . . . . . . . . . . . . . . . . . .

Schipperges, Heinrich: Goethe - seine Kunst zu leben (Grosche) $\ldots \ldots \ldots \ldots \ldots \ldots$

Schmiedebach, Heinz-Peter: Robert Remak (1815-1865). Ein jüdischer Arzt im Spannungsfeld von Wissenschaft und Politik (Mörgeli) ..........................

Schnalke, Thomas: Diseases in wax. The history of the medical moulage (Leven) . . . . 322

Seils, Markus: Friedrich Albrecht Carl Gren in seiner Zeit 1760-1798 (Ledermann) . . . 144

Soemmerring, Samuel Thomas: Briefwechsel, 1761/65 - Oktober 1784 (Steinke) . . . . . 144

Soemmerring, Samuel Thomas: Rezensionen für die Göttingischen gelehrten Anzeigen.

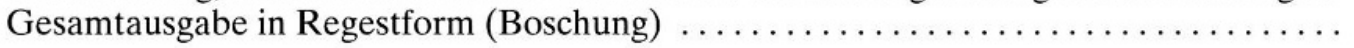

Veröffentlichungen aus dem Archiv zur Geschichte der Max-Planck-Gesellschaft (Glaus) 145

Waardt, J. M. D. de: Voedselvoorschriften in boeteboeken (Huizink) . . . . . . . . . 147

Walter, Bernd: Psychiatrie und Gesellschaft in der Moderne (Müller) . ........... 323

Weiss, Burghard: «Forschungsstelle D». Der Schweizer Ingenieur Walter Dällenbach (1892-1990), die AEG und die Entwicklung kernphysikalischer Grossgeräte im national-

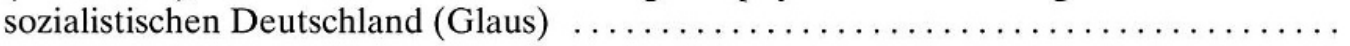


Willi-Hangartner, Regula: Zur Geschichte des Apothekenwesens im Kanton Schwyz

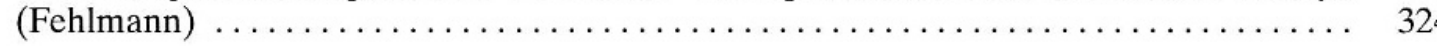

Wilmanns, Juliane C.: Der Sanitätsdienst im Römischen Reich (Frei) … . . . . . . . 149

Die Wisenberg-Panoramen von Samuel Birmann (1813) und Peter Schmid-Ruosch (1990)

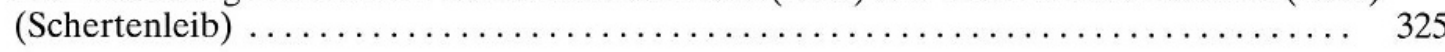

Wolf, Rudolf (Wolfiana): Rudolf Wolfs Jugendtagebuch 1835-1841 (Ineichen) $\ldots \ldots \ldots \quad 150$

Woodward, John; Jütte, Robert (eds.): Coping with sickness. Historical aspects of health

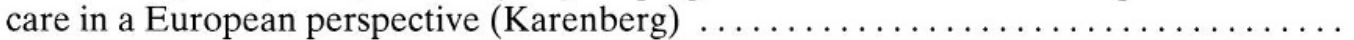

